\title{
Exact Gravitational Field of the Infinitely Long Rotating Hollow Cylinder
}

\author{
ECKART FREHLAND \\ Lehrstuhl für Theoretische Physik der Universität Konstanz
}

Received December 13, 1971

\begin{abstract}
The vacuum line element inside an infinitely long rotating hollow cylinder is the usual flat space line element. It is fitted in a most general way to the general cylindrical vacuum field outside at the singular hypersurface $R_{0}=$ const, representing the infinitely thin hollow cylinder. With the use of the jump conditions at $R_{0}=$ const the surface densities $\tau_{\mu}^{v}$, of which the energy-momentum-stress tensor $\tau_{\mu}^{v}$ of the shell consists, are calculated. The physical properties of the cylinder, as derived from the eigenvalues and -vectors of $\tau_{\mu}^{v}$, and the generated gravitational field are discussed in full detail.
\end{abstract}

\section{Introduction}

Recently we have shown [1] (in the following cited as I), that the general stationary cylindrical vacuum field, found by Davies and Caplan [2] is static, whereafter, it is identical with Levi-Civitas general static solution [3]. Hence any stationary (rotating) cylindrical matter distributions generate a static cylindrical vacuum field. As far as we know the only rigorously treated example for this class of matter distributions is the rotating cylinder of Van Stockum [4], consisting of incoherent matter.

In this paper we present the general solution for the uniformly rotating infinitely thin hollow cylinder. The general-relativistic procedure of constructing the gravitational field of such surface distributions has been given by Lanczos [5], Israel [6], Treder [7] et al. The main results, which we shall need in this paper, are: Choosing natural (Gaussian) coordinates in which the metric tensor is continuous across the (singular) hypersurface $x_{1}=a=$ const, we get the line-element in the form

$$
d s^{2}=-d x^{1^{2}}+g_{i k} d x^{i} d x^{k} \quad(i, k=2,3,4) .
$$

The energy-momentum-stress tensor $T_{\mu}^{v}$ has the surface-density structure ${ }^{1}$

$$
T_{\mu}^{v}=\tau_{\mu}^{v} \delta\left(x_{1}-a\right) .
$$

According to the definition of the $\delta$-function Einstein's field equations of gravitation

$$
R_{\mu v}=-\left(T_{\mu v}-\frac{1}{2} T g_{\mu \nu}\right)
$$

${ }^{1}$ Greek indices run from 1-4, latin indices (except $i, k$ ) from 1-3. 
yield

$$
\begin{aligned}
\left(\tau_{i k}-\frac{1}{2} \tau g_{i k}\right)_{\left(x^{1}=a\right)} & =\frac{1}{2}\left(\frac{\partial}{\partial x^{1}} g_{i k}\right)_{\left(x^{1}-a\right) \rightarrow+a}-\frac{1}{2}\left(\frac{\partial}{\partial x^{1}} g_{i k}\right)_{\left(x^{1}-a\right) \rightarrow-0} \\
\tau_{1 \alpha} & =0 .
\end{aligned}
$$

In order to apply this method to the rotating hollow cylinder, we shall proceed as follows: Firstly, we join continuously the vacuum solutions inside and outside the shell in the most general way. In I we have shown that, if the interior field is free of singularities, the spacetime inside the cylinder is (at least locally) flat, distinguishing three topologically different cases. Here we shall restrict ourselves to the case of the simply connected spacetime inside. Secondly, we calculate the surface densities $\tau_{\mu}^{v}$ and determine the eigenvalues and eigenvectors of $\tau_{\mu}^{\nu}$, which describe invariantly the physical properties of the shell.

The results are discussed in Chapter 3. We find particularly that the sum of the eigenvalues of $\tau_{\mu}^{v}$ vanishes necessarily, i.e. $\tau_{\mu}^{v}$ is tracefree. This implies the nonexistence of $a$ rotating hollow cylinder consisting of incoherent matter.

Finally we investigate the gravitational effects caused by the rotation of the cylinder. Gravitational effects of rotating bodies were first regarded by Thirring [8] in 1918. Since that time many other contributions about this subject have been published, out of which we cite only a few [9-18]. However in all these papers approximation methods are used. In contrast to this we discuss in the following the infinitely long rotating hollow cylinder rigorously. Certainly this example is far away from being physical, but it is interesting under the aspect of the Thirring effect, which is coming out particulary clear.

\section{The Fitting Procedure}

The general cylindrical line element satisfying the vacuum field equations is according to $\mathrm{I},(3.2)$

$$
\begin{aligned}
d s^{2}= & -\left(d R^{2}+R^{4\left(\frac{A+1}{A^{2}+3}\right)} d \Phi^{2}+R^{2\left(\frac{A^{2}-1}{A^{2}+3}\right)} d Z^{2}\right) \\
& +R^{4\left(\frac{1-A}{A^{2}+3}\right)} d T^{2} .
\end{aligned}
$$

The condition of finiteness of the eigenvalues of the Riemann tensor at $R=0$ causes for the field inside the cylinder the restriction on the cases $A= \pm 1, A= \pm \infty$; hence (2.1) results in:

$$
\begin{array}{ll}
A=+1: & d s^{2}=-\left(d R^{2}+R^{2} d \mathscr{*}^{2}+d \mathscr{Z}^{2}\right)+d \stackrel{*}{T}^{2}, \\
A=-1: & d s^{2}=-\left(d R^{2}+d \stackrel{*}{\Phi}^{2}+d \mathscr{Z}^{2}\right)+R^{2} d \stackrel{*}{T}^{2}, \\
A= \pm \infty: & d s^{2}=-\left(d R^{2}+d \stackrel{*}{\Phi}^{2}+R^{2} d \stackrel{*}{Z}^{2}\right)+d \stackrel{*}{T}^{2} .
\end{array}
$$


Herein the asterisks denote the coordinates appropriate to the interior line element.

In (2.1) and (2.2) the variables $\Phi, \stackrel{*}{\Phi}$ are regarded as generalized angle coordinates. So we still have to introduce for $\Phi, \stackrel{*}{\Phi}$ the periods $P, \stackrel{*}{P}$. The remaining coordinates have the following regions of validity: $-\infty \leqq T\left({ }^{*}\right)$ $\leqq+\infty,-\infty \leqq Z\left(Z_{Z}^{*}\right) \leqq+\infty$ and $R$ according to Table 1 . This determines the different topological structure of spacetimes given by $(2.2 \mathrm{a}-\mathrm{c})$. In the cases $A=-1, A= \pm \infty$ the circumference of the 2-dimensional spacelike hypersurfaces $T=$ const, $R=$ const (cylinders) is independent of $R$. In case of $A=+1$ it is proportional to $R$.

We shall discuss only the case $A=+1$. This restriction is required by the condition that the spacetime is simply connected. Obviously, for $A=+1$ (cf. (2.2 a)) we must choose the period $\stackrel{*}{P}$ to be $2 \pi$, in order to avoid a singular structure at $R=0$. In contrast to this for $A=-1$ and $A= \pm \infty$ the spacetimes are not simply connected and $R=0$ does not define an axis of rotation. However we wish to point out that the whole fitting procedure, presented in this paper, could analogously be performed also for these cases.

In the following we will join continuously the fields (2.2a) inside and (2.1) outside at the singular hypersurface $R=R_{0}$ defining the hollow cylinder of radius $R_{0}$.

With the transformations

$$
\begin{aligned}
& d \Phi=d \hat{\Phi} \cdot R_{0}^{\left(\frac{(1-A)^{2}}{A^{2}+3}\right)}, \quad d Z=d \hat{Z} \cdot R_{0}^{-\left(\frac{A^{2}-1}{A^{2}+3}\right)} \\
& d T=d \hat{T} \cdot R_{0}^{-2\left(\frac{1-A}{A^{2}+3}\right)}
\end{aligned}
$$

the line element (2.1) (outside) takes the form

$$
\begin{aligned}
d s^{2}= & -\left(d R^{2}+R^{2}\left(\frac{R}{R_{0}}\right)^{-2\left(\frac{(1-A)^{2}}{A^{2}+3}\right)} d \hat{\Phi}^{2}\right. \\
& \left.+\left(\frac{R}{R_{0}}\right)^{2\left(\frac{A^{2}-1}{A^{2}+3}\right)} d \hat{Z}^{2}\right)+\left(\frac{R}{R_{0}}\right)^{4\left(\frac{1-A}{A^{2}+3}\right)} d \hat{T}^{2} .
\end{aligned}
$$

If we identify the coordinates $R, \stackrel{*}{*}, \stackrel{*}{Z}, \stackrel{*}{T}$ and $R, \hat{\Phi}, \hat{Z}, \hat{T}$, the connection with (2.2a) gives, as we shall see, the static case of the non-rotating hollow cylinder. Hence in the general non static (stationary) case we have to perform a coordinate transformation before the connection of $(2.2 \mathrm{a})$ and (2.4).

Global cylindrical symmetry of the field implies the existence of appropriate coordinates, in which the metric tensor is dependent on the radial distance $R$ alone. These properties are given by the line element 
Period of the angle coordi-

nate

a) $R, \stackrel{*}{\Phi}, \stackrel{*}{Z}, \stackrel{*}{T} \quad \underline{R \leqq R_{0}}: \quad d s^{2}=-\left(d R^{2}+R^{2} d \stackrel{*}{\Phi^{2}}+d \stackrel{*}{Z}^{2}\right)+d \stackrel{*}{T}^{2}$

$$
\begin{aligned}
& \underline{R \geqq R_{0}:} d s^{2}=-\left\{d R^{2}+\left[\left(1+b^{2} R_{0}^{2}\right) R^{2}\left(\frac{R}{R_{0}}\right)^{-2\left(\frac{(1-A)^{2}}{A^{2}+3}\right)}-b^{2} R_{0}^{4}\left(\frac{R}{R_{0}}\right)^{4\left(\frac{1-A}{A^{2}+3}\right)}\right] d \Phi^{2} \quad 0 \leqq R<R_{0}\left(\frac{1+b^{2} R^{2}}{b^{2} R_{0}^{2}}\right)^{\left(\frac{A^{2}+3}{8|A|}\right)}\right. \\
& -2\left(1+b^{2} R_{0}^{2}\right)^{\frac{1}{2}} b\left[R^{2}\left(\frac{R}{R_{0}}\right)^{-2\left(\frac{(1-A)^{2}}{A^{2}+3}\right)}-R_{0}^{2}\left(\frac{R}{R_{0}}\right)^{4\left(\frac{1-A}{A^{2}+3}\right)}\right] d \stackrel{*}{\Phi} \cdot d \stackrel{*}{T} \\
& \left.+\left(\frac{R}{R_{0}}\right)^{2\left(\frac{A^{2}-1}{A^{2}+3}\right)} d \stackrel{*}{Z}^{2}\right\}+\left[\left(1+b^{2} R_{0}^{2}\right)\left(\frac{R}{R_{0}}\right)^{4\left(\frac{1-A}{A^{2}+3}\right)}-b^{2} R^{2}\left(\frac{R}{R_{0}}\right)^{-2\left(\frac{(1-A)^{2}}{A^{2}+3}\right)}\right] d \stackrel{*}{T}^{2} \quad 0 \leqq \stackrel{*}{\Phi} \leqq 2 \pi
\end{aligned}
$$

b) $R, \hat{\Phi}, \hat{Z}, \hat{T} \quad R \leqq R_{0}: \quad d s^{2}=-\left(d R^{2}+d \hat{Z}^{2}\right)-\left[\left(1+b^{2} R_{0}^{2}\right) R^{2}-b^{2} R_{0}^{4}\right] d \hat{\Phi}^{2}-2 b\left(1+b^{2} R_{0}^{2}\right)^{\frac{1}{2}}\left(R_{0}^{2}-R^{2}\right) d \hat{\Phi} d \hat{T}$ $+\left[\left(1+b^{2} R_{0}^{2}\right)-b^{2} R^{2}\right] d \hat{T}^{2}$

$\underline{R \geqq R_{0}}: \quad d s^{2}=-\left\{d R^{2}+R^{2}\left(\frac{R}{R_{0}}\right)^{-2\left(\frac{(1-A)^{2}}{A^{2}+3}\right)} d \hat{\Phi}^{2}+\left(\frac{R}{R_{0}}\right)^{2\left(\frac{A^{2}-1}{A^{2}+3}\right)} d \hat{Z}^{2}\right\}+\left(\frac{R}{R_{0}}\right)^{4\left(\frac{1-A}{A^{2}+3}\right)} d \hat{T}^{2}$

c) $R, \tilde{\Phi}, \tilde{Z}, \tilde{T} \quad \underline{R \leqq R_{0}}: \quad d s^{2}=-\left(d R^{2}+R^{2} d \tilde{\Phi}^{2}+d \tilde{Z}^{2}\right)+2-\frac{b R^{2}}{\left(1+b^{2} R_{0}^{2}\right)^{\frac{1}{2}}} d \tilde{\Phi} d \tilde{T}+\left(1-\left(\frac{b^{2}}{1+b^{2} R_{0}^{2}}\right) R^{2}\right) d \tilde{T}^{2}$

$A \geqq 0:$

$$
\begin{aligned}
& \underline{R \geqq R_{0}}: \quad d s^{2}=-\left\{d R^{2}+\left[\left(1+b^{2} R_{0}^{2}\right) R^{2}\left(\frac{R}{R_{0}}\right)^{-2\left(\frac{(1-A)^{2}}{A^{2}+3}\right)}-b^{2} R_{0}^{4}\left(\frac{R}{R_{0}}\right)^{4\left(\frac{1-A}{A^{2}+3}\right)}\right] d \tilde{\Phi}^{2}\right. \\
& \left.-2 \frac{b}{\left(1+b^{2} R_{0}^{2}\right)^{\frac{1}{2}}} R_{O}^{2}\left(\frac{R}{R_{0}}\right)^{4\left(\frac{1-A}{A^{2}+3}\right)} d \tilde{\Phi} d \tilde{T}+\left(\frac{R}{R_{0}}\right)^{2\left(\frac{A^{2}-1}{A^{2}+3}\right)} d \tilde{Z}^{2}\right\} \\
& +\left(\frac{1}{1+b^{2} R_{0}^{2}}\right)\left(\frac{R}{R_{0}}\right)^{4\left(-\frac{1-A}{A^{2}+3}\right)} d \tilde{T}^{2}
\end{aligned}
$$

$0 \leqq R \leqq+\infty$

$\frac{A \leqq 0:}{0 \leqq R} \leqq R_{0}\left(\frac{1+b^{2} R_{0}^{2}}{b^{2} R_{0}^{2}}\right)^{\left(\frac{A^{2}+3}{8|A|}\right)}$

$0 \leqq \tilde{\Phi} \leqq 2 \pi$ 
outside (cf. (2.4)) in the coordinates $R, \hat{\Phi}, \hat{Z}, \hat{T}$ as well as inside (cf. (2.2a)) in the coordinates $R, \stackrel{*}{\Phi}, \stackrel{*}{Z}, \stackrel{*}{T}$. In order to preserve these properties the coordinate transformations between $\frac{*}{\Phi}, T^{*}$ and $\hat{\Phi}, \hat{T}$ must be linear ${ }^{2}$. Taking into acount the condition of continuity of $g_{\mu v}$ at $R=R_{0}$, we get:

$$
\begin{array}{ll}
\stackrel{*}{Z}=\hat{Z} & \stackrel{*}{\Phi}=-b \hat{T}+\left(1+b^{2} R_{0}^{2}\right)^{\frac{1}{2}} \hat{\Phi} \\
& \stackrel{*}{T}=\left(1+b^{2} R_{0}^{2}\right)^{\frac{1}{2}} \hat{T}-b R_{0}^{2} \hat{\Phi}
\end{array}
$$

with the inversion

$$
\hat{Z}=\stackrel{*}{Z} \quad \begin{aligned}
& \hat{\Phi}=b \stackrel{*}{T}+\left(1+b^{2} R_{0}^{2}\right)^{\frac{1}{2}} \stackrel{*}{\Phi} \\
& \\
& \hat{T}=\left(1+b^{2} R_{0}^{2}\right)^{\frac{1}{2}} \stackrel{*}{T}+b R_{0}^{2} \stackrel{*}{\Phi} .
\end{aligned}
$$

In the coordinates $R, \stackrel{*}{\Phi}, \stackrel{*}{Z}, \stackrel{*}{T}$ and $R, \hat{\Phi}, \hat{Z}, \hat{T}$ the region of validity of $R$ does not extend from $R=0$ to $R=+\infty$ (see Table 1). For $A \geqq 0$ we can introduce further coordinates $R, \tilde{\Phi}, \tilde{Z}, \tilde{T}$, valid in the whole region $0 \leqq R \leqq+\infty$, which are given by:

$$
\tilde{\Phi}=\tilde{\Phi}-\frac{b}{\left(1+b^{2} R_{0}^{2}\right)^{\frac{1}{2}}} \cdot \tilde{T} \quad \begin{aligned}
& \stackrel{*}{T}=\tilde{T} \\
& \stackrel{*}{Z}=\tilde{Z}
\end{aligned}
$$

As to be seen from (2.4) and (2.3) or Table 1 the gravitational field of the hollow cylinder is dependent on the three parameters $A, b$ and $R_{0}$, corresponding to the three physical properties of the cylinder, i.e. the surface density of matter, the rotational velocity and the radius. The relations between $A, b$ and these properties are established in the following through the jump-conditions (1.4).

\section{The Physical Properties of the Hollow Cylinder}

The invariant physical properties of the hollow cylinder are determined by investigation of the eigenvalues and eigenvectors of $\tau_{\mu}^{v}$. If we regard the timelike normalized eigenvektor $u^{\mu}$ as the 4-velocity of the mass elements of the cylinder, the eigenvalue $\mu$ belonging to $u^{\mu}$ is the proper (surface) density of matter. The three eigenvalues to the three orthogonal spacelike eigenvectors (orthogonal to $u^{\mu}$ ) mean the stresses occuring in the cylindrical shell. As to be seen from the general relations (1.4) the eigenvalue to that spacelike eigenvektor, being orthogonal to the hypersurface $R_{0}=$ const, vanishes, i.e. the stresses are lying in the shell, as expected from the surface density structure of matter.

We emphasize that this interpretation of the eigenvalues and eigenvectors is unquestionably not necessary but the most simple one. It includes a certain (e.g. elastic) structure of matter of the cylinder. Heat flow for instance is excluded. But nevertheless the eigenvalues and

\footnotetext{
${ }^{2} \mathrm{~A} Z$-dependent transformation is not admissible for symmetry reasons.
} 
-vectors contain invariant informations which are independent of the special model of matter being regarded. Hence the results of this chapter are valid in general, only the expressions "surface density of matters", "motion" etc. are related to a special model and could be replaced by other names.

\section{a) The Static Case}

First we discuss the most simple case $b=0$, in which the coordinates $\stackrel{*}{\Phi}, \hat{\Phi}, \tilde{\Phi}$ and $\stackrel{*}{T}, \hat{T}, \tilde{T}$ are identical (cf. (2.5)). The surface densities $\tau_{\mu \nu}$ follow from (1.4) in view of the linelement of Table 1 to be

$$
\begin{aligned}
\tau_{44} & =2\left(\frac{1-A}{A^{2}+3}\right) \frac{1}{R_{0}} \\
\tau_{22} & =\left(\frac{(1-A)^{2}}{A^{2}+3}\right) R_{0}, \quad \tau_{33}=\left(\frac{1-A^{2}}{A^{2}+3}\right) \frac{1}{R_{0}} . \\
\tau_{\mu \nu} & =0 \text { otherwise }
\end{aligned}
$$

The normalized eigenvectors of (3.1) are

$$
u^{\mu}=\delta_{4}^{\mu}, \quad b_{(a)}^{\mu}=\frac{\delta_{a}^{\mu}}{\sqrt{-g_{a a}}}
$$

with the nonzero eigenvalues

$$
\begin{aligned}
\mu & =\tau_{4}^{4}=2\left(\frac{1-A}{A^{2}+3}\right) \frac{1}{R_{0}} \\
\sigma_{(\Phi)} & =\tau_{2}^{2}=-\left(\frac{(1-A)^{2}}{A^{2}+3}\right) \frac{1}{R_{0}}, \quad \sigma_{(Z)}=\tau_{3}^{3}=\left(\frac{A^{2}-1}{A^{2}+3}\right) \frac{1}{R_{0}} .
\end{aligned}
$$

According to $u^{\mu}$ (cf. (3.2)) the mass elements of the cylinder are at rest relative to the reference system, defined by the hypersurface orthogonal time-like normal vector field $v^{\mu}\left(x^{\alpha}\right)=\frac{\delta_{4}^{\mu}}{\sqrt{g_{44}}}$. In this sense we characterize the cylinder to be non-rotating.

With the relations (3.3) we can express in (2.4) (outside) the metric tensor by the radius $R_{0}$, the surface density $\mu$ and the stresses $\sigma_{(\Phi)}, \sigma_{(Z)}$. Hence the linelement inside and outside of the cylinder is:

$$
\begin{aligned}
\underline{0 \leqq R \leqq R_{0}}: \quad d s^{2}= & -\left(d R^{2}+R^{2} d \mathscr{\Phi}^{2}+d Z^{2}\right)+d \stackrel{*}{T}^{2} \\
\underline{R_{0} \leqq R \leqq+\infty}: d s^{2}= & -\left(d R^{2}+R^{2}\left(\frac{R}{R_{0}}\right)^{2 \sigma_{(\Phi)} R_{0}} d \mathscr{\Phi}^{2}\right. \\
& \left.+\left(\frac{R}{R_{0}}\right)^{2 \sigma_{(Z)} R_{0}} d Z^{2}\right)+\left(\frac{R}{R_{0}}\right)^{2 \mu R_{0}} d \stackrel{*}{T}^{*} .
\end{aligned}
$$


Apart from the factor $2 \pi$ the dimensionless quantities $\mu R_{0}, \sigma_{(\Phi)} R_{0}$, $\sigma_{(Z)} R_{0}$ describe line densities with respect to the $Z$-axis. Hence the limit case of the infinitely long $\operatorname{rod}\left(R_{0}=0\right)$ is included, taking the line element in the form (2.1). From (3.3) we find the following relations between the eigenvalues of $\tau_{\mu}^{\nu}{ }^{3}$ :

$$
\begin{gathered}
\mu+\sigma_{(\Phi)}+\sigma_{(Z)}=0 \\
\left(\mu R_{0}\right)^{2}+\left(1+\sigma_{(\Phi)} R_{0}\right)^{2}+\left(\sigma_{(Z)} R_{0}\right)^{2}=1 .
\end{gathered}
$$

Thus hold the restrictions:

$$
\begin{aligned}
& -\frac{1}{3} \leqq \mu R_{0} \leqq+1 \\
& -\frac{1}{3} \leqq \sigma_{(Z)} R_{0} \leqq+1 \\
& -\frac{4}{3} \leqq \sigma_{(\Phi)} R_{0} \leqq 0
\end{aligned}
$$

Therefore solutions for the non-rotating infinite hollow cylinder exist only for mass densities in the interval $-\frac{1}{3} \leqq \mu R_{0} \leqq 1$. However for physical reasons the mass density may be restricted to positive values.

\section{b) The General Stationary Case}

In the general case $b \neq 0$ the nonvanishing components of $\tau_{\mu v}$ following from (1.4) with the use of the linelement Table 1, line a) are ${ }^{4}$ :

$$
\begin{aligned}
& \tau_{44} \stackrel{*}{=} 2\left(\frac{1-A}{A^{2}+3}\right) \frac{1}{R_{0}}-b^{2} R_{0}^{2}\left(\frac{4 A}{A^{2}+3}\right) \frac{1}{R_{0}} \\
& \tau_{24} \stackrel{*}{=}-b R_{0}\left(1+b^{2} R_{0}^{2}\right)^{\frac{1}{2}}\left(\frac{4 A}{A^{2}+3}\right) \\
& \tau_{22} \stackrel{*}{=}-b^{2} R_{0}^{2}\left(\frac{4 A}{A^{2}+3}\right) R_{0}+\left(\frac{(1-A)^{2}}{A^{2}+3}\right) R_{0} \\
& \tau_{33} \stackrel{*}{=}\left(\frac{1-A^{2}}{A^{2}+3}\right) \frac{1}{R_{0}} .
\end{aligned}
$$

It is easy to see, that as in the static case holds:

$$
\tau_{\mu}^{\mu}=0
$$

Furthermore the spacelike eigenvectors $b_{(R)}^{\mu}$ and $b_{(Z)}^{\mu}$ are the same as in the static case, cf. (3.2). For finding the remaining eigenvectors $b_{(\Phi)}^{\mu}$ and $u^{\mu}$ we make instead of solving the eigenvalue equations for $\tau_{\mu \nu}$ the following ansatz for the timelike normalized eigenvector $u^{\mu}$, which is suggested

${ }^{3}$ (3.5) are just the relations known from the Kasner solution (see I, (3.3)).

$4 \stackrel{*}{=}$ indicates, that the equation is valid only in the coordinates $R, \stackrel{*}{\Phi}, \stackrel{*}{Z}, \stackrel{*}{T}$. 
by the Minkowskian structure of the interior region:

$u^{1} \stackrel{*}{=} u^{3} \stackrel{*}{=} 0, \quad u^{2} \stackrel{*}{=} \frac{\Omega}{\sqrt{1-\beta^{2}}}, \quad u^{4} \stackrel{*}{=} \frac{1}{\sqrt{1-\beta^{2}}}, \quad|\beta|=|\Omega| R_{0}<1$.

According to this the condition of orthogonality yields for the normalized eigenvector $b_{(\Phi)}^{\mu}$ :

$$
b_{(\Phi)}^{1} \stackrel{*}{=} b_{(\Phi)}^{3} \stackrel{*}{=} 0, \quad b_{(\Phi)}^{2} \stackrel{*}{=} \frac{1}{R_{0} \sqrt{1-\beta^{2}}}, \quad b_{(\Phi)}^{4} \stackrel{*}{=} \frac{\beta}{\sqrt{1-\beta^{2}}} .
$$

Herein the parameter $\beta$ has the following simple invariant meaning: it is the absolute value of the 3 -velocity of the mass elements of the cylinder with respect to the inertial system in the flat space inside defined by

$$
v^{\mu} \stackrel{*}{=} \delta^{4 \mu} \text {. }
$$

The eigenvalue $\sigma_{(Z)}$, describing the stress in $Z$-direction, is in view of (3.7) as in the static case:

$$
\sigma_{(Z)}=\left(\frac{A^{2}-1}{A^{2}+3}\right) \frac{1}{R_{0}} .
$$

Using the ansatz (3.8) the eigenvalue equations of $\tau_{\mu \nu}$ result in:

$$
\begin{gathered}
\mu=-b\left(1+b^{2} R_{0}^{2}\right)^{\frac{1}{2}}\left(\frac{4 A}{A^{2}+3}\right) \beta+\frac{2}{R_{0}}\left(\frac{1-A}{A^{2}+3}\right)-b^{2} R_{0}\left(\frac{4 A}{A^{2}+3}\right) \\
\mu \beta=b^{2} R_{0}\left(\frac{4 A}{A^{2}+3}\right) \beta-\frac{1}{R_{0}}\left(\frac{(1-A)^{2}}{A^{2}+3}\right) \beta+b\left(1+b^{2} R_{0}^{2}\right)^{\frac{1}{2}}\left(\frac{4 A}{A^{2}+3}\right) \\
\sigma_{(\Phi)}=b^{2} R_{0}\left(\frac{4 A}{A^{2}+3}\right)-\frac{1}{R_{0}}\left(\frac{(1-A)^{2}}{A^{2}+3}\right)+b\left(1+b^{2} R_{0}^{2}\right)^{\frac{1}{2}}\left(\frac{4 A}{A^{2}+3}\right) \beta \\
\sigma_{(\Phi)} \beta=-b\left(1+b^{2} R_{0}^{2}\right)^{\frac{1}{2}}\left(\frac{4 A}{A^{2}+3}\right)+\frac{2}{R_{0}}\left(\frac{1-A}{A^{2}+3}\right) \beta-b^{2} R_{0}\left(\frac{4 A}{A^{2}+3}\right) \beta .
\end{gathered}
$$

The relations (3.10) and (3.11) represent the reduction of $A$ and $b$ on the physical quantities $\mu, R_{0}, \beta, \sigma_{(\Phi)}$ and $\sigma_{(Z)}$; one finds:

with

$$
\begin{gathered}
A^{2}=\frac{1+3 \sigma_{(Z)} R_{0}}{1-\sigma_{(Z)} R_{0}}, \\
b^{2} R_{0}^{2}=\frac{1}{2}\left[\left(1+4 \frac{\beta^{2}}{\left(1-\beta^{2}\right)^{2}}\left(\mu-\sigma_{(\Phi)}\right)^{2} R_{0}^{2} S^{-1}\right)^{\frac{1}{2}}-1\right]
\end{gathered}
$$

$$
S=\left(1+\sigma_{(Z)} R_{0}-3 \sigma_{(Z)}^{2} R_{0}^{2}\right) .
$$


Apart from the special case $\mu=\sigma_{(\Phi)}$ (cf. Chapter $3 \mathrm{c}$ ) it follows from (3.12b) that $b=0$ (static case) exactly if $\beta=0$ (non rotating cylinder).

On the other hand only three physical properties of the cylinder are freely available, e.g. $\mu, R_{0}$ and $\beta$. The five relations (3.10) and (3.11), between which the dependence ( 3.7 a) exists, allow to determine uniquely the dependent four quantities $A, b, \sigma_{(\Phi)}, \sigma_{(Z)}$. Thus one finds in addition to the Eqs. (3.12a) and (3.12b):

$$
\begin{gathered}
\sigma_{(\Phi)} R_{0}=-\frac{1}{2}\left(\mu R_{0}+\frac{1}{1-\beta^{2}}\right)\left[1 \pm\left(1-\frac{4 \mu R_{0}\left(\mu R_{0}-\frac{\beta^{2}}{1-\beta^{2}}\right)}{\left(\mu R_{0}+\frac{1}{1-\beta^{2}}\right)^{2}}\right)\right] \\
\sigma_{(Z)}=-\left(\mu+\sigma_{(\Phi)}\right) .
\end{gathered}
$$

Herewith also the validity of the ansatz (3.8 a) has been shown.

We emphasize that the passage to the limit $R_{0}=0$ in (3.12) and (3.13) can be performed (as in the static case) so that the line element (2.1) describes the field of an infinitely long rod with spin. Finally we note, that (3.13) agrees with (3.5) in the static case $b=0$.

\section{c) Detailed Discussion}

In Fig. 1 the dimensionless quantities $\sigma_{(\Phi)} R_{0}, \sigma_{(Z)} R_{0}$ are drawn as functions of $\mu R_{0}$ for different values of $\beta$. The four shaded sectors I $(0 \leqq A \leqq 1)$, II $(+1 \leqq A \leqq+3)$, III $(+3 \leqq A \leqq+\infty)$ and IV $(A \leqq 0)$ are the regions, in which solutions for the stationary rotating hollow cylinder exist.

First, we discuss the physically most interesting sector I with $\mu R_{0} \geqq 0,-\frac{1}{3} \leqq \sigma_{(\Phi)} R_{0} \leqq+\frac{1}{6}$ (see also Fig. 2). The sign of the stress term $\sigma_{(\Phi)} R_{0}$ depends on the rotational speed $\beta$ and the mass density $\mu$. It is negative, if the "gravitational attractive forces" are greater than the "centrifugal forces" and inverse. According to the relation (3.13a) $\sigma_{(\Phi)}$ vanishes for $\mu R_{0}=\left(\frac{\beta^{2}}{1-\beta^{2}}\right)$, i.e. the two forces compensate each other. For $|\beta| \geqq \frac{1}{2}$ the centrifugal force dominates for all possible values of $\mu R_{0}$. It is remarkable, that for the mass density $\mu R_{0}$ exists an upper limit depending on the rotational speed $\beta$. It varies between $+\frac{2}{3}(\beta=0)$ and $+\frac{1}{3}(\beta \rightarrow 1)$; all physically relevant densities ly far below this limit.

The behavior of negative rotating masses (sectors II, III) is completely different from that of positive masses. The tangential stress term $\sigma_{(\Phi)} R_{0}$ is negative for all possible values of $\mu$ and $\beta$. Hence, as to be expected, the centrifugal forces are directed inward as well as the gravitational forces. 


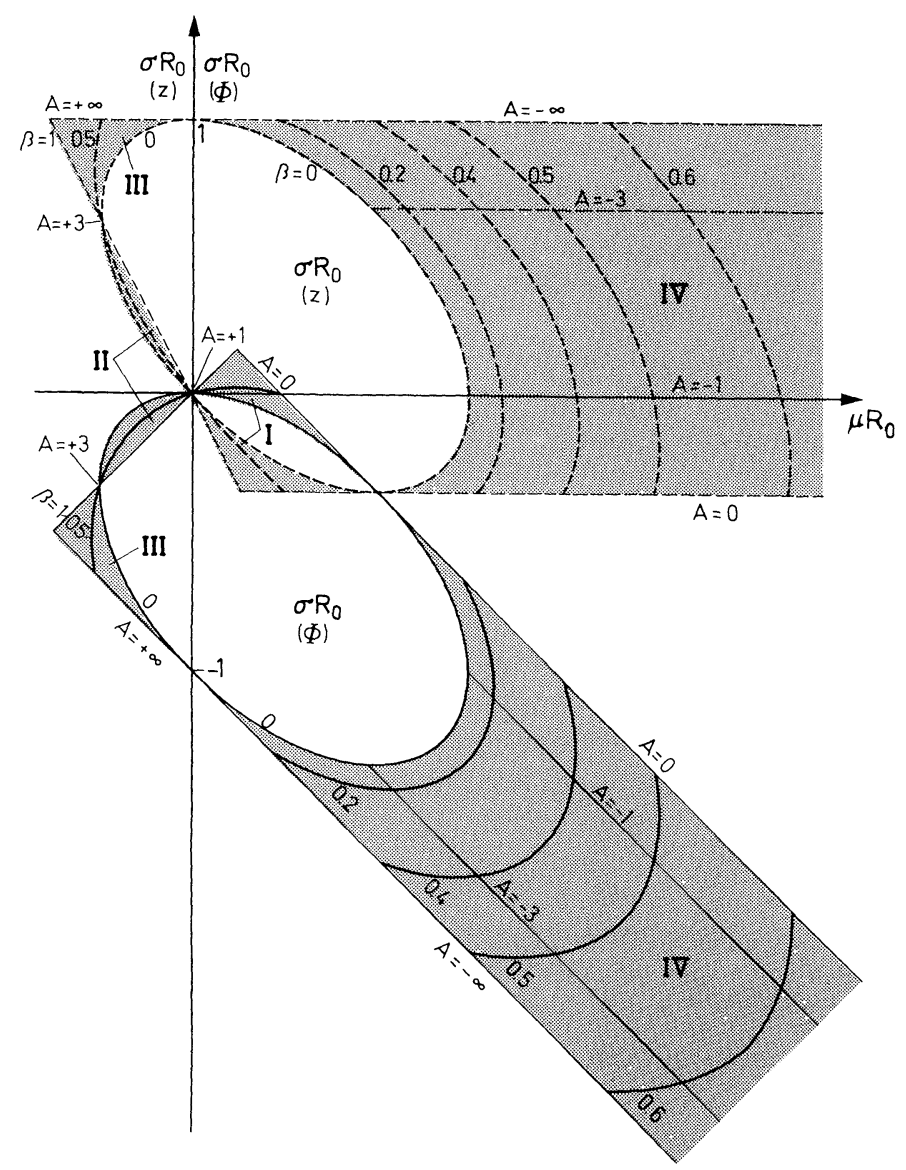

Fig. 1. The stresses $\sigma_{(\Phi)}$ and $\sigma_{(\Phi)}$ as functions of $\mu$ for different values of the rotational speed $\beta$

Furthermore in Fig. 1 we have marked some special values of $A$. For the cases $A=+1$ and $A=+3$ the lines $A=$ const degenerate into points, and the eigenvalues of $\tau_{v}^{\mu}$ are independent of $\beta$. While the case $A=+1$ results in the flat space with vanishing $\tau_{v}^{\mu}$ and $b=0$, in the case $A=+3$ the Riemann tensor (outside the cylinder) is of degenerate type D (see I). Particularly we get from (3.12) and (3.13) $\mu R_{0}=\sigma_{(\Phi)} R_{0}=-\frac{1}{3}$ and from (3.12b) $b=0$ as well as for $A=+1$. Thus the gravitational field and the nonvanishing eigenvalues of $\tau_{v}^{\mu}$ are independent of the rotation $\beta$ of the cylinder. However this curious case $A=+3$ lies in a physically non relevant region.

Sector IV seems to be of less interest. For $\beta \rightarrow 1$ we find $\mu R_{0},\left|\sigma_{(\Phi)} R_{0}\right| \rightarrow \infty$. We renounce to a further discussion of this sector. 


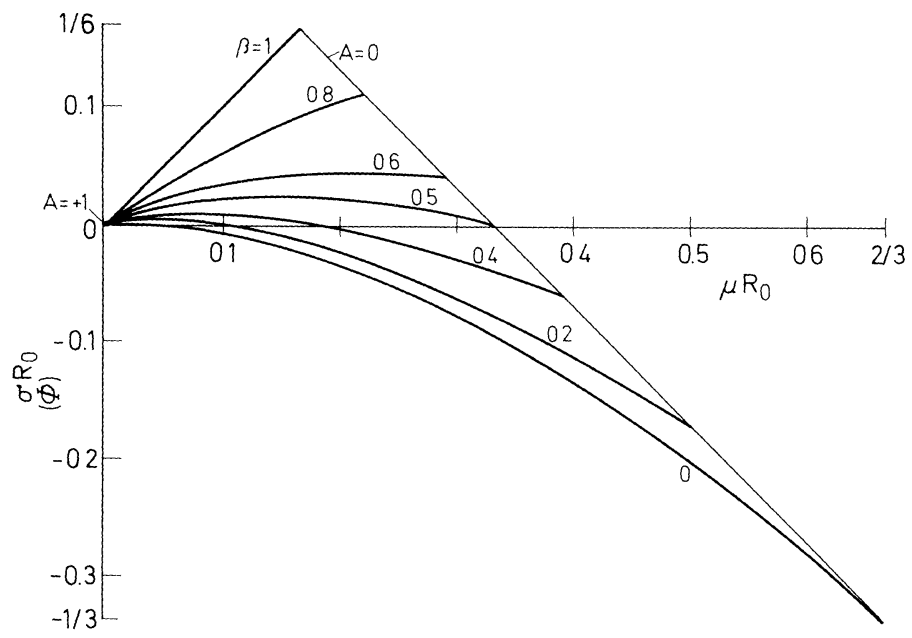

Fig. 2. The course of the tangential stresses in sector I

Finally we give in case of the sectors I and II approximate values of $\sigma_{(\Phi)}$ and $\sigma_{(Z)}$ for small mass densities $\mu R_{0}$. We get from (3.13):

$$
\sigma_{(\Phi)} \approx \mu \beta^{2}, \quad \sigma_{(Z)} \approx-\mu\left(1+\beta^{2}\right) .
$$

In [18] we have calculated the energy-momentum-stress tensor of the hollow cylinder in special relativistic approximation. (3.14) agrees with these results, if we choose the constant of integration $\sigma^{Z Z}$ occuring in [18], (2.2.6) to be $-\left(1+\beta^{2}\right) \mu$. The value of $\sigma_{(\Phi)}$ in (3.14) represents the usual centrifugal term.

\section{Thirring Effect}

From (2.1) and (2.2) follows immediately that the field is static inside as well as outside the cylinder, i.e. it admits inside and outside a hypersurface orthogonal timelike Killing field $\eta^{\mu}$ and $\xi^{\mu}$ respectively:

$$
\begin{aligned}
& \eta^{\mu} \stackrel{*}{=} \delta_{4}^{\mu}, \\
& \xi^{\mu} \cong \delta_{4}^{\mu} .
\end{aligned}
$$

Both fields are continuous across the singular hypersurface $R_{0}=$ const. But the vector field $\eta^{\mu}$ is hypersurface orthogonal only inside and $\xi^{\mu}$ only outside the cylinder. The hypersurfaces are $\stackrel{*}{T}=$ const and $\hat{T}=$ const respectively. As it is easy to see, the gravitational field does not admit (for $b \neq 0$ ) a timelike Killing field, which goes continuously across the 
surface $R_{0}=$ const and is hypersurface orthogonal inside as well as outside. This behavior of the field of the rotating cylinder can be given the following physical interpretation: We define two reference frames (one inside and one outside) by the congruences of the timelike worldlines given by (4.1). The tangent normal vector fields to these congruences are:

and

$$
v^{\mu} \stackrel{*}{=} \delta_{4}^{\mu} \quad \text { for } \quad R \leqq R_{0}
$$

$$
w^{\mu} \hat{=} \frac{\delta_{4}^{\mu}}{\sqrt{g_{44}}} \text { for } R \geqq R_{0} .
$$

Using Table 1 and (2.5) we find for the absolute value of the 3-dimensional relative velocity $v_{\text {rel. }}$ at $R=R_{0}$ between the reference frames given by $w^{\mu}$ and $v^{\mu}$ :

$$
v_{\text {rel. }}=\omega R_{0}=\frac{b R_{0}}{\left(1+b^{2} R_{0}^{2}\right)^{\frac{1}{2}}} .
$$

$\omega$ is the angular velocity between the two reference frames. This effect, which was first established in linear approximation by Thirring [8]; is known as the "dragging of inertial frames by rotating bodies", which causes the appearance of "Coriolis (magnetic-type) forces" [19].

From (4.3) follows that $\operatorname{sign} \omega=\operatorname{sign} b$ and $\omega=0$ if and only if $b=0$. Hence the Thirring effect is an immediate consequence of the rotation of the cylinder. From (3.12) and (4.3) we find $\omega R_{0}$ as a function of the physical quantities:

$$
\begin{gathered}
\left|\omega R_{0}\right|=\left(\frac{\alpha-1}{\alpha+1}\right) \\
\alpha=\left[1+\frac{4 \beta^{2}}{\left(1-\beta^{2}\right)^{2}}\left(\mu-\sigma_{(\Phi)}\right)^{2} R_{0}^{2} S^{-1}\right]^{\frac{1}{2}} .
\end{gathered}
$$

For the sign of $\omega$ one gets:

$$
\operatorname{sign} \omega=\operatorname{sign}\left(\beta \cdot\left(\mu-\sigma_{(\Phi)}\right) R_{0} \cdot A\right) .
$$

\section{Final Discussion}

In Fig. 3 we have drawn $\omega R_{0}\left(\mu R_{0}\right)$ for different values of the rotational speed $\beta$. The main results, as to be seen from (4.4) and Fig. 3, are:

The maximum value $\left|\omega R_{0}\right|=1$, i.e. $\alpha=+\infty$, is reached for the limiting cases of maximum rotational speed

$$
|\beta|=1 \quad \text { and } \quad A=0 \quad\left(\sigma_{(Z)} R_{0}=-\frac{1}{3}, S=0\right),
$$

i.e. maximum (negative) stresses in $Z$-direction (see also Fig. 1).

The sign of $\omega$ is according to (4.5) determined by the direction of the rotational speed $\beta$, the sign of $\left(\mu-\sigma_{(\Phi)}\right) R_{0}$ and $A$. Hence in sector I 


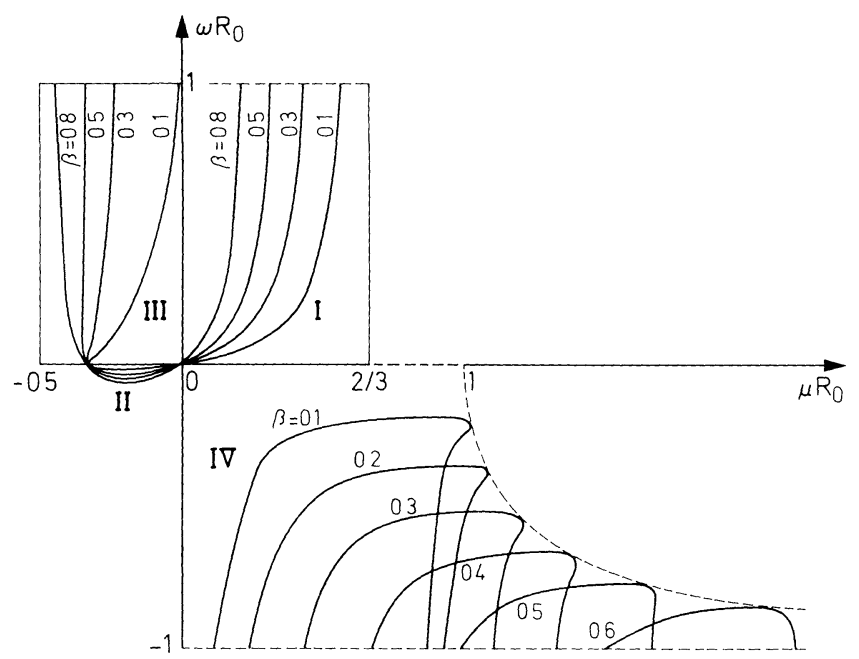

Fig. 3. The relative rotation $\omega R_{0}$ of the reference frames $v^{\mu}$ and $w^{\mu}$ as a function of the mass density for different values of $\beta$

of Fig. $1\left(1 \geqq A \geqq 0, \mu R_{0} \geqq 0, \sigma_{(\Phi)} R_{0} \leqq \mu R_{0}\right)$ the reference system outside, given by $w^{\mu}$, rotates with respect to the reference frame $v^{\mu}$ inside in the same direction as the cylinder. In sector II (negative mass density, $\left.\left(\mu-\sigma_{(\Phi)}\right) R_{0} \leqq 0,3 \geqq A \geqq 1\right)$ it rotates in opposition to the cylinder as well as in sector IV $\left(\mu R_{0} \geqq 0,\left(\mu-\sigma_{(\Phi)}\right) R_{0} \geqq 0, A \leqq 0\right)$, while in sector III $\left(+\infty \geqq A \geqq 3, \mu R_{0}<0\right.$ but $\left.\left(\mu-\sigma_{(\Phi)}\right) R_{0} \geqq 0\right)$ the absolute value of the stress term $\sigma_{(\Phi)} R_{0}$ predominates $\mu R_{0}$ and we get a behavior as in sector I.

A consequence of the Thirring effect is, that observers in the reference frame $w^{\mu}$ outside, placed nearly at $R=R_{0}$, will measure a rotational speed $\beta_{(\alpha)}$ of the mass elements of the cylinder, which is different from the speed $\beta$ measured by the observers in the reference frame $v^{\mu}$ inside. Performing the same procedure as described in Chapter (3.b) in the coordinates $R, \hat{\Phi}, \hat{Z}, \hat{T}$ we finally get the following relation between $\beta$ and $\beta_{(x)}$.

$$
\frac{\beta}{\left(1-\beta^{2}\right)}=\left(\frac{4 A}{A^{2}+3}\right) \frac{\beta_{(\alpha)}}{\left(1-\beta_{(\alpha)}^{2}\right)} .
$$

The sign of $\beta$ and $\beta_{(\alpha)}$ is the same in the sectors I-III of Fig. $1(A \geqq 0)$, but opposite in sector IV $(A \leqq 0)$. Together with (4.4) this yields the curious result that for $A<0$ the relative rotation of the frames $v^{\mu}$ and $w^{\mu}$ is "faster" than the rotation of the cylinder.

I thank Prof. H. Dehnen for discussions. This work was supported by the Deutsche Forschungsgemeinschaft. 


\title{
References
}

1. Frehland, E.: The general stationary gravitational vacuum field of cylindrical symmetry. Commun. math. Phys. 23, 127-131 (1971).

2. Davies, H., Caplan, T.A.: The space-time metric inside a rotating cylinder. Proc. Cambridge Philos. Soc. 69, 325-327 (1971).

3. Levi-Civita, T.: $d s^{2}$ einsteiniani in campi newtoniani. Nine notes in Rend. Reala Accad. Lincei Ser. 5ª 26 (1917), 27 (1918), 28 (1919).

4. van Stockum, W.J.: The gravitational field of a distribution of particles rotating about an axis of symmetry. Proc. Roy. Soc. Edinburgh 57, 135-154 (1937).

5. Lanczos, C.: Flächenhafte Verteilung der Materie in der Einsteinschen Gravitationstheorie. Ann. Physik 74, 518 (1924).

6. Israel, W.: Singular hypersurfaces and thin shells in general relativity. Nuovo Cimento $44 \mathrm{~B}, 1-14$ (1966), 48 B, 463 (1967).

7. Treder,H.J.: Flächenbelegungen und Topologie in der allgemeinen Relativitätstheorie. Ann. Physik 24, $234-242$ (1970).

8. Thirring, H.: Über die Wirkung rotierender ferner Massen in der Einsteinschen Gravitationstheorie. Phys. Z. 19, 33-39 (1918); 22, 29-30 (1921).

9. Lense, J., Thirring, H.: Über den Einfluß der Eigenrotation der Zentralkörper auf die Bewegung der Planeten und Monde nach der Einsteinschen Gravitationstheorie. Phys. Z. 19, 156-163 (1918).

10. van Stockum, W.J.: The precession of the inertial frame of a rotating body. Proc. Roy. Irish Acad. Sect. A, 44, 109-122 (1938).

11. Clark, G.L.: The external gravitational and electromagnetic fields of rotating bodies. Proc. Roy. Soc. A 201, 488-509 (1950).

12. Bass, L., Pirani,F.A.E.: On the gravitational effects of distant rotating masses. Phil. Mag. 40, 850-856 (1955).

13. Hönl, H., Maue, A. W.: Über das Gravitationsfeld rotierender Massen. Z. Physik 144, 152-167 (1956).

14. - Soergel-Fabricius, Ch.: Coriolis-Kräfte im Einstein-Kosmos und das Machsche Prinzip. Z. Physik 163, 571-581 (1961).

15. Levy, H., Robinson, W.J.: The rotating body problem. Proc. Cambridge Philos. Soc. 60, 279-285 (1963).

16. Brill, D. R., Cohen,J.M.: Rotating masses and their effect on inertial frames. Phys. Rev. 143, 1011-1015 (1965).

17. Teyssandier,P.: Contribution à l'étude des masses quasisphériques tournantes en relativité génerale. Ann. Inst. Henri Poincaré 12, 263-283 (1970).

18. Frehland, E.: Energy-momentum-stress tensor and gravitational field of uniformly rotating masses. Int. J. Theoret. Phys. 4, 33-40 (1971).

19. Hönl,H., Dehnen,H.: Allgemein-relativistische Dynamik und Machsches Prinzip. Z. Physik 191, 313-328 (1966).

\author{
E. Frehland \\ Universität Konstanz \\ D-7750 Konstanz \\ Postfach 733 \\ Germany
}

